\title{
Clinicopathological Profile of Metastatic Cervical Lymph Nodes- A Descriptive Study in a Tertiary Care Centre
}

\author{
Authors \\ Dr Praveen N. ${ }^{1}$, Dr. Swathilal. S. A. ${ }^{2}$, Dr Lakshmanan. P. P. ${ }^{3}$, Dr Deeps. C. K. ${ }^{4}$ \\ ${ }^{1}$ Associate Professor of Plastic Surgery, Medical College, Thiruvananthapuram \\ ${ }^{2}$ Assistant professor of E. N. T. Medical College Calicut \\ ${ }^{3}$ Additional Professor of Surgery Medical College Calicut \\ ${ }^{4}$ Assistant Professor, COVAS Pookot
}

\begin{abstract}
The single most important factor determining the survival of patients with head and neck malignancy is the presence or absence of metastatic disease in the neck. clinicopathological profile of metastatic lymph nodes were conducted in the department of surgery, Medical College, Kozhikkode for a three years. Thirty nine cases of head and neck malignancy having either clinically evident cervical metastasis or those with a high chance of occult metastasis were selected. Twenty six of 39(66.7\%) were male with male to female ratio 2.1 The mean age was 58.7(10.7) years. Most of the patients in this study belonged to 61-70 year group. The most common site of the primary lesion was at the anterior tongue(41\%).Twenty one (53.8\%) patients was at stage II, followed by 11(28.2\%) patients in the stage III. The most common symptom was ulcer of the oral cavity (60\%) followed by neck swelling in 15\%. Cervical nodes were pathologically positive for metastasis in twenty one of the thirty nine cases (53.8\%) of these seven were unilateral and four were bilateral. The commonest presenting feature was ulcer in the mouth followed by neck swelling,pain in the mouth, difficulty in swallowing, change in voice and ankyloglossia. The present study revealed most of the lesion were of ulcerative type.
\end{abstract}

\section{Introduction}

The single most important factor determining the survival of patients with head and neck malignancy is the presence or absence of metastatic disease in the neck ${ }^{1}$. Unfortunately medical opinion is divided on various aspects of metastatic neck disease. ${ }^{2}$ Controversies remain in assessment of neck disease, the prophylactic treatment of metastatic nodes, place of radical neck dissection as opposed to modified radical neck dissection and the role of radiotherapy ${ }^{3}$.

Accurate determination of a neck node requires exacting clinical examination combined with considered use of appropriate supportive investigational diagnostic techniques ${ }^{4}$. Clinical examination of neck lacks strict objectivity being affected by various factors like the thickness of patient's neck skin, the amount of subcutaneous fat and the width obscured by the sternomastoid muscle ${ }^{5}$. But the potential benefits of clinical examination far outweigh these drawbacks and it still remains as the most important method of assessing regional lymph nodes ${ }^{6}$.

Impact of various aspects of primary disease in determining the neck metastasis is of utmost importance. A consensus needs to be reached on 
which ones are the more important determinants of metastasis; so that appropriate management of the neck can be planned ${ }^{7}$.

Since its first description by George Washington Crile in 1906, classical radical neck dissection has undergone an increasing number of modifications $^{8}$. It has now come to selective neck dissection in which only those lymph node groups are removed that are more likely to contain metastatic deposits from the concerned primary. ${ }^{9,10}$. But its rationale and indications remain controversial. Many critics view it as only a staging procedure; with the need for a more comprehensive dissection if obvious metastic nodes are present on histological examination. The objective of this study was to describe the clinicopathological profile of metastatic lymph nodes.

\section{Materials and Methods}

This is descriptive study was conducted in the department of surgery, Medical College, Kozhikkode during 2007 to 2010.

Patients were recruited into the study only after the approval of the protocol by the research and ethics committee in the institution. Only those patients giving informed consent were recruited to the study. In addition, we conducted the study conforming to declaration of Helsinki in all phases of the study. This particular study was a part of a larger study comparing the diagnostic accuracy of clinical, sonological and histopathological examination of metastatic lymph node in the neck.

A total of thirty nine patients with head and neck malignancy having either clinically evident cervical metastasis or those with a high chance of occult metastasis admitted in various wards of department of surgery and radiotherapy were included in the study. They were treated with wide local excision of the primary combined with neck dissection with or without reconstruction of the primary area. All consecutive patients satisfying the inclusion and exclusion criteria were included in the study. We included only patients above the age of 13. Those patients with history of preoperative radiotherapy were excluded from the study. Moreover we excluded those patients with recurrent swelling and previous neck dissection. A formal sample size calculation was done.

Thirty nine cases of head and neck malignancy having either clinically evident cervical metastasis or those with a high chance of occult metastasis were selected. Proper history was elicited and clinical examination was performed. Neck examination was carried out standing behind the patient. Using both the hands, each side of the slightly flexed neck was palpated simultaneously in a sequential manner starting from the submental and submandibular triangles, then the neck anterior to the sternomastoid passing from above downwards, the supraclavicular fossa, then upwards into the posterior triangle and forwards across the sternomastoids to the nodes of the anterior triangle which are palpated a second time. Ultrasound examination was done in the N0 neck and in cases were deeper structure fixity was suspected. CT scan was carried out as indicated as per the standard treatment protocol.

The patients were then posted for surgery. After the neck dissection the specimen was cut according to the level of lymph nodes. The specimen were fixed in $10 \%$ buffered formalin and sent for histopathology examination where they were embedded in paraffin wax. Similarly the specimen of the primary lesions were fixed and sent. Tissue sections were stained with haematoxylin-eosin. The primary lesions were studied for the type and grade of malignancy and the presence or absence of perineural or lymphovascular invasion. Lymph nodes were studied for the presence or absence of metastasis and extracapsular spread.

All data were taken in a well designed case report form. All these data were abstracted into an excel based database and then analyzed.

All data were analyzed with Epiinfo 6.0 software and R statistical software. Contiguous data were summarized with mean and standard deviation or median and interquartile range depending on the distribution of the data. All categorical data were summarized with percentages and number. 


\section{Results}

Of the patients included in this study,26 of $39(66.7 \%)$ were male with male to female ratio 2.1 The mean age was 58.7(10.7) years. Most of the patients in this study belonged to 61-70 year group. The most common site of the primary lesion was at the anterior tongue $(41 \%)$ as shown in the figure1.

Figure 1: site of primary lesion.

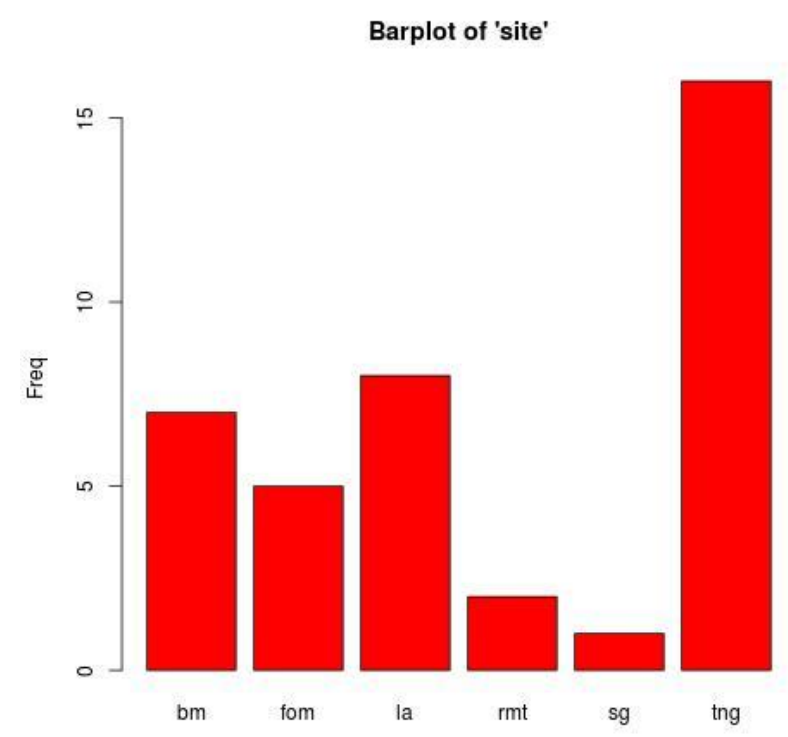

\# bm-buccalmucosa, fom-floor of mouth, la-lower alveolus, rmt-retromolartrigone, sg-supraglottis, tng-anterior tongue.

In this study, 21(53.8\%) patients was at stage II, followed by $11(28.2 \%)$ patients in the stage III. The baseline characteristics of the study participants are summarized in the table 1 . The most common symptom was ulcer of the oral cavity $(60 \%)$ followed by neck swelling in $15 \%$, other complaints being pain, ankyloglossia, dysphagia and change in voice(figure 2). Five patients $(12.8 \%)$ had hypertension while three (7.6\%) were diabetics. Sixteen of the study population $(41 \%)$ were smokers. Fifteen patients (38.4\%) were addicted to panchewing. Of these six were both smokers and panchewers. One patient was an alcoholic.
Table 1: baseline characteristics of

\begin{tabular}{ll} 
the patients & {$[$ ALL] } \\
Variables & $\mathrm{N}=39$ \\
\hline Age & $58.7(10.7)$ \\
$\begin{array}{l}\text { Sex: } \\
\text { Female }\end{array}$ & $13(33.3 \%)$ \\
Male & $26(66.7 \%)$ \\
site: & \\
Buccal mucosa & $7(17.9 \%)$ \\
Floor of mouth & $5(12.8 \%)$ \\
Lower alveolus & $8(20.5 \%)$ \\
Retromolartrigone & $2(5.13 \%)$ \\
supraglottis & $1(2.56 \%)$ \\
Anterior tongue & $16(41.0 \%)$
\end{tabular}

Thirty three of the thirty nine patients $(84.6 \%)$ had clinically palpable cervical node; while six (15.4\%) were clinically node negative. Among the clinically positive group thirty (91\%) had unilateral cervical metastasis, while three $(9 \%)$ had bilateral cervical metastasis. The most common lymph node level affected was level $\mathrm{Ib}$ (51.2\%) followed by II (34.9\%) whereas the least commonly affected were level IV and V (2.3\%) as shown in table 2.Analysis of stage of neck nodes revealed that most patients were in the $\mathrm{N} 1$ stage (18/39) 46.1\%, twelve were N2, six were N0 and only three were N3.

Analysis of morphology of the primary lesion revealed that in most cases it was ulcerative $(51.2 \%)$, it was ulcer proliferative in $38.5 \%$ and proliferative in $10.3 \%$.In $58.9 \%$ (23/39) of the cases the primary lesion was $\mathrm{T} 2$, while in $30.8 \%$ (12/39) it was T4. Two patients each were having $\mathrm{T} 1$ and $\mathrm{T} 3$ tumors.

Ultrasound of the neck was done in ten patients. All the six patients with NO neck clinically were found to be $\mathrm{N} 0$ after ultrasound examination also. All the six patients with N0 neck clinically were found to be $\mathrm{N} 0$ after ultrasound examination also. In one patient with clinically N3 unilateral node, ultrasound detected nodes on the opposite side, while in another patient with N3 node, ultrasound detected multiple nodes on the same side. One of the two patients with $\mathrm{N} 1$ neck in whom ultrasound was done had multiple nodes on the same side while in the other ultrasound confirmed the clinical finding of single unilateral node. All the thirty nine cases were squamous cell carcinoma of 
which twenty $(51.3 \%)$ were moderately differentiated, sixteen (41.0\%) were well differentiated and only three $(7.7 \%)$ were poorly differentiated. Lymphovascular invasion was present in four $(10.3 \%)$ cases while it was absent in the rest thirty five $(89.7 \%)$.

Figure 2: stage of disease grouped by histopathology

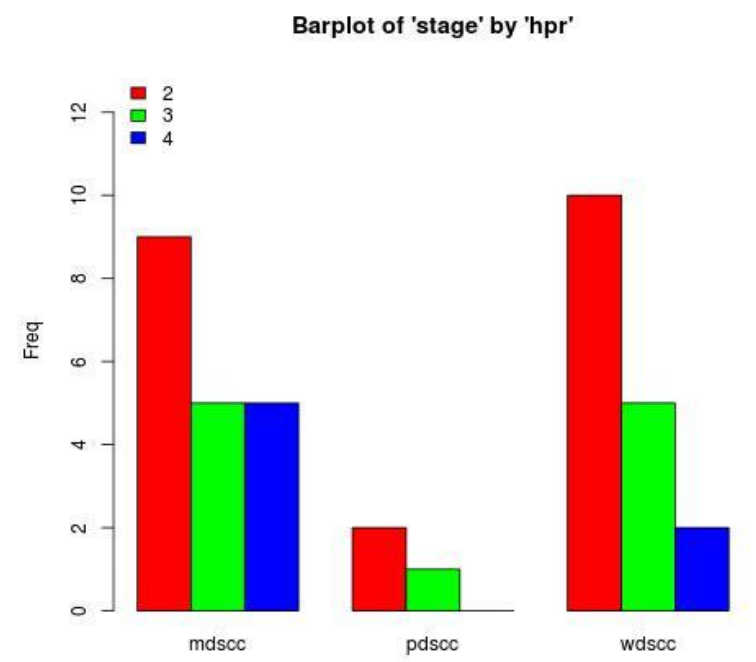

\#mdcc-moderately differentiated SCC, pdsccpoorly differentiated SCC, wdscc-widely differentiated SCC

Cervical nodes were pathologically positive for metastasis in twenty one of the thirty nine cases $(53.8 \%)$ of these seven were unilateral and four were bilateral and were N0 and only three were N3.Most common level of node affected by metastasis was level II which was positive in seventeen neck dissections (75\%) either singly or in combination with nodes at other levels. This was followed by level $\mathrm{Ib}$ which was positive in twelve neck dissections. The least affected was level Ia which was involved in only four cases. In no case metastasis bypassed level I, II or III to involve level IV or V. Extracapsular involvement was present in seven of the twenty one pathologically positive neck dissections while it was absent in the rest.Five of the six clinically negative neck were negative pathologically also, while the other patient with T2 tongue lesion had metastasis at level Ib and II. Nine of the eighteen
$\mathrm{N} 1$ and four of the twelve clinically N2 patients were pathologically negative for metastasis. But all the clinically N3 were pathologically positive.

\section{Discussion and Conclusion}

Undetected nodal metastasis is the commonest cause of treatment failure in head and neck cancer. Hence, regional control of nodal metastasis in the neck is an important factor in predicting the clinical course and outcome of the patient with head and neck malignancy (Hibbert et al., 1983)21. The oral cavity has a rich lymphatic drainage. Palpable nodes on presentation reduces the survival by about one half46. In the present study thirty eight out of the total thirty nine patients had primary malignancy in the oral cavity.

There is a progressive increase in the incidence of cancer with age, with the elderly showing a much increased rate of susceptibility ${ }^{11}$. This is believed to be related to a protracted conditioning period that is associated with environmental factors, especially tobacco chewing or smoking.

The present study also reflects the same pattern with maximum cases in the sixth and seventh decade and a mean age of 58.7 years. Shah et at., (1990)40 found the mean age to be 60 years in patients with oral cancer $^{12,13}$. The mean age of patients presenting with oral cancer in Kerala was found to be 57.8 years by Sankaranarayanan et al., (1992)36 in an earlier study ${ }^{14}$.

As evident from table, the incidence of oral cancer in this study is more among males, the male female ratio being $2: 1$. The results are comparable with previous studies. Hamner et al., (1986)20 found the male female ratio of oral cancer to be $2: 1^{15}$. Studies by Cherian et al., (1991)9 and Sankaranarayanan et al., (1992)36 also showed a higher incidence in males ${ }^{16}$. This is probably due to males being more habituated to tobacco chewing, smoking and alcohol consumption when compared to women.

Most of the patients with oral cancer present with ulcer. However, a few present with a metastatic node in the neck with no complaints related to the oral cavity ${ }^{11}$. 
In the present study too, the commonest presenting feature was ulcer in the mouth followed by neck swelling. Pain in the mouth, difficulty in swallowing, change in voice and ankyloglossia were the other symptoms with which the patients presented.

Brunn et al., (1976)6 showed the average delay between the start of symptom and treatment to be 10.5 months $^{17}$.

In present study, the duration of symptoms ranged from one month to three years with a mean duration of 7.67 months. This reduction in the mean duration reflects increased awareness about this health problem among the population of our state.

Smoking and alcohol are the two most strongly implicated factors in the aetiology of oral cancer 18,19. The increased incidence of oral cancer in some parts of our country is related to the habit of pan chewing and reverse smoking prevalent in those parts ${ }^{20}$.

The present study also reflects a similar epidemiological pattern with $66 \%$ of the people addicted to smoking, pan chewing or alcohol.

In oral cancer, majority of the nodes on presentation or which develop subsequently, are ipsilateral and are confined to the submandibualr or upper and mid jugular groups. Contralateral or bilateral nodes are more likely in lesions which are close to the midline e.g. in the floor of the mouth and/or dorsum of the tongue ${ }^{11}, 39,41$. Metastatic disease in the lower neck may occur in the absence of obvious disease higher in the neck; particularly with primary tongue lesions ${ }^{21}$ Woolgar et al., 199753).

In the present study the most common lymph node level affected on clinical examination was found to be level Ib followed by level II and the least commonly affected were level IV and V. This finding is similar to that reported in world literature.

Various studies have shown tongue to be the most common site of oral cancer followed by floor of mouth and lower alveolus ${ }^{11}$.

In the present study too the most common site of primary lesion was anterior tongue followed by lower alveolus, buccal mucosa, floor of mouth and retromolartrigone, in the decreasing order of frequency.

The vast majority of mouth cancers are both ulcerative and infilitrative; exophytictumours are less common ${ }^{22}$. In this study too most of the lesion were of ulcerative type.

\section{Acknowledgment}

I would like to thank Dr.I.P.Yadev for his help in the analysis of the data and constant encouragement.

\section{Reference}

1. Martin H. Cancer of the head and neck. Journal of the American Medical Association 1948; 137(16): 1366-76.

2. Garg M, Beitler JJ. Controversies in management of the neck in head and neck cancer. Current treatment options in oncology 2004; 5(1): 35-40.

3. Kowalski LP, Sanabria A. Elective neck dissection in oral carcinoma: a critical review of the evidence. Acta Otorhinolaryngologica Italica 2007; 27(3): 113-7.

4. Sakaguchi T, Yamashita Y, Katahira K, et al. Differential diagnosis of small round cervical lymph nodes: comparison of power Doppler US with contrast-enhanced CT and pathologic results. Radiation medicine 2001; 19(3): 119-25.

5. Anand N, Chaudhary N, Mittal MK, Prasad R. Comparison of the efficacy of clinical examination, ultrasound neck and computed tomography in detection and staging of cervical lymph node metastasis in head and neck cancers. Indian Journal of Otolaryngology and Head \& Neck Surgery 2007; 59(1): 19-23.

6. Kallalli B, Rawson K, Kumari V, Patil S, Singh A, Sulaga S. Comparison between clinical examination, ultrasonography, and computed tomography in assessment of cervical lymph node metastasis in oral squamous cell carcinoma. Journal of 
Indian Academy of Oral Medicine and Radiology 2016; 28(4): 364-9.

7. Dralle H, Musholt TJ, Schabram J, et al. German Association of Endocrine Surgeons practice guideline for the surgical management of malignant thyroid tumors. Langenbeck's Archives of Surgery 2013; 398(3): 347-75.

8. Carlson ER, Cheung A, Smith B, Pfohl C. Neck dissections for oral/head and neck cancer: 1906-2006. Journal of Oral and Maxillofacial Surgery 2006; 64(1): 4-11.

9. Ferlito A, Rinaldo A, Silver CE, et al. Elective and therapeutic selective neck dissection. Oral oncology 2006; 42(1): 1324.

10. Samant S, Robbins KT. Evolution of neck dissection for improved functional outcome. World journal of surgery 2003; 27(7): 805-10.

11. Kerr AG. Scott-Brown's Otolaryngology No-4Rhinilogy: London: Butter worth, 1987; 1987.

12. Shah JP, Candela FC, Poddar AK. The patterns of cervical lymph node metastases from squamous carcinoma of the oral cavity. Cancer 1990; 66(1): 109-13.

13. Carvalho AL, Singh B, Spiro RH, Kowalski LP, Shah JP. Cancer of the oral cavity: a comparison between institutions in a developing and a developed nation. Head \& neck 2004; 26(1): 31-8.

14. Sankaranarayanan R, Nair MK, Mathew B, Balaram P, Sebastian P, Dutt SC. Recent results of oral cancer research in Kerala, India. Head \& neck 1992; 14(2): 107-12.

15. Hamner J. Aetiology and epidemiology of oral cancer. Cancer and the Oral Cavity 1986: 17-30.

16. Kuriakose M, Sankaranarayanan M, Nair $\mathrm{MK}$, et al. Comparison of oral squamous cell carcinoma in younger and older patients in India. European Journal of
Cancer Part B: Oral Oncology 1992; 28(2): 113-20.

17. Bruun JP. Time lapse by diagnosis of oral cancer. Oral surgery, oral medicine, oral pathology 1976; 42(2): 139-49.

18. Silverman S, Griffith M. Smoking characteristics of patients with oral carcinoma and the risk for second oral primary carcinoma. The Journal of the American Dental Association 1972; 85(3): 637-40.

19. Smith EM. An analysis of cohort mortality from tongue cancer in Japan, England and Wales and the United States. International journal of epidemiology 1982; 11(4): 32935.

20. Reddy CR. Carcinoma of hard palate in India in relation to reverse smoking of chuttas. Journal of the National Cancer Institute 1974; 53(3): 615-9.

21. Droulias C, Whitehurst J. The lymphatics of the tongue in relation to cancer. The American surgeon 1976; 42(9): 670.

22. Gleeson M. Scott-Brown's Otorhinolaryngology: Head and Neck Surgery 7Ed: 3 volume set: CRC Press; 2008. 